\title{
Collisions of low-energy antiprotons with molecular hydrogen: ionization, excitation and stopping power.
}

\begin{abstract}
Armin Lühr · Alejandro Saenz
Received: date / Accepted: date

Abstract A time-dependent coupled-channel approach ' was used to calculate ionization, excitation, and energyloss cross sections as well as energy spectra for antiproton and proton collisions with molecular hydrogen for impact energies $8<E<4000 \mathrm{keV}$.
\end{abstract}

Keywords antiproton · hydrogen molecule · collision . stopping power

PACS $34.50 . \mathrm{Bw} \cdot 34.50 . \mathrm{Gb}$

\section{Introduction}

A large amount of theoretical work has been done for antiproton $(\bar{p})$ collisions with He atoms stimulated by ' discrepancies between experiment and theory lasting for more than a decade [7. In the case of hydrogen also a number of contributions exist for atomic targets whereas the knowledge on low-energy $\bar{p}$ collisions with the simplest two-electron molecule $\mathrm{H}_{2}$ is rather ' poor [1,5], especially, concerning theory [2,11. Precise data on $\bar{p}+\mathrm{H}_{2}$ are, however, of great interest in many fields. They can, e.g., be used to determine the stopping power which is a prerequisite for the design of low-energy $\bar{p}$ storage rings taking the interactions with residual-gas atoms and molecules into account. But also for the preparation of accurate future experiments with low-energy $\bar{p}$, which in turn can be used as a test of competing theoretical approaches, the maximum of the stopping power is of importance. A short overview on the present status of $\bar{p}+\mathrm{H}_{2}$ shall be given.

Institut für Physik, AG Moderne Optik, Humboldt-Universität zu Berlin, Hausvogteiplatz 5-7, D-10117 Berlin, Germany.

Tel.: +49-(0)30-2093-4814

E-mail: Armin.Luehr@physik.hu-berlin.de
The used method has already been discussed in some detail elsewhere 10,11,12 as well as partly in the article on interactions of antiprotons with alkali-metal atoms [13. A time-dependent close-coupling approach is employed which uses the impact-parameter method. The target molecule is treated as an effective one-electron system by applying a model potential 12,16. The wave function of the collision process is expanded in a onecenter approach in eigenfunctions of the one-electron model Hamiltonian of the target. Thereby, the radial part is expanded in B-spline functions and the angular part in a symmetry-adapted sum of spherical harmonics.

\section{Results}

Ionization In Fig. 1 the present $\mathrm{H}_{2}$ results for ionization and electron loss by $\bar{p}$ and $p$ impact, respectively, are compared with cross sections given in the literature and results for atomic hydrogen multiplied by two. It can be seen that the naive picture of the $\mathrm{H}_{2}$ molecule as being practically the same as two independent $\mathrm{H}$ atoms only holds at high impact energies $E$. For high and intermediate impact energies the present $\bar{p}$ results agree with the experiments by Anderson et al. 11 and Hvelplund et al. [5] although they are slightly lower than the measured data. At low energies $E<$ $20 \mathrm{keV}$, however, the experimental results are significantly smaller than the present calculations which partly can be explained with experimental problems not accounted for at that time [6]. These problems also occurred for $\bar{p}+$ He cross sections measured with the same apparatus [5] and were identified by very recent He measurement [7]. The other theoretical curve [2] describes the $\bar{p}+\mathrm{H}_{2}$ cross section only for $E>200 \mathrm{keV}$ 


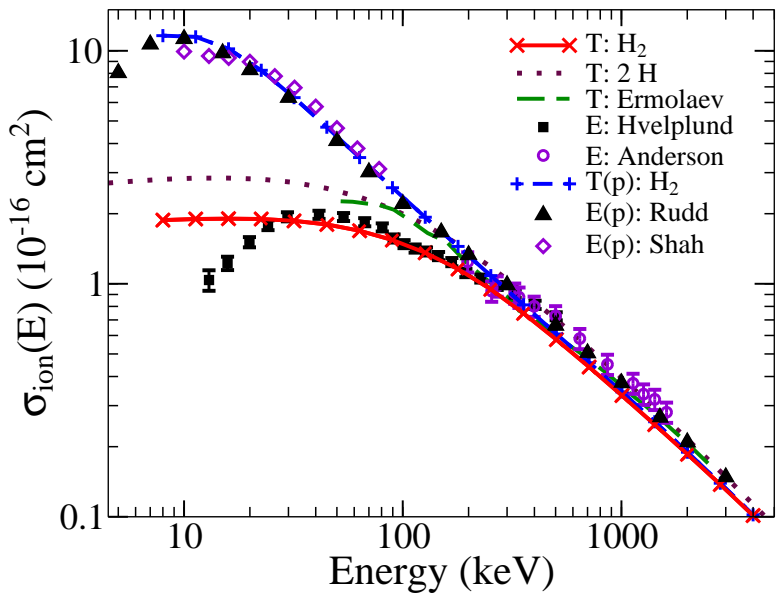

Fig. 1 Ionization cross section. Theory. $\bar{p}$ : solid curve, present; dashed curve, Ermolaev; dotted curve, two times atomic H. $p$ : dash-dotted curve, present. Experiment. $\bar{p}$ : squares, Hvelplund et al. [5]; circles, Anderson et al. 1]. $p$ : triangles, Rudd et al. [14];diamonds, Shah et al. [15].

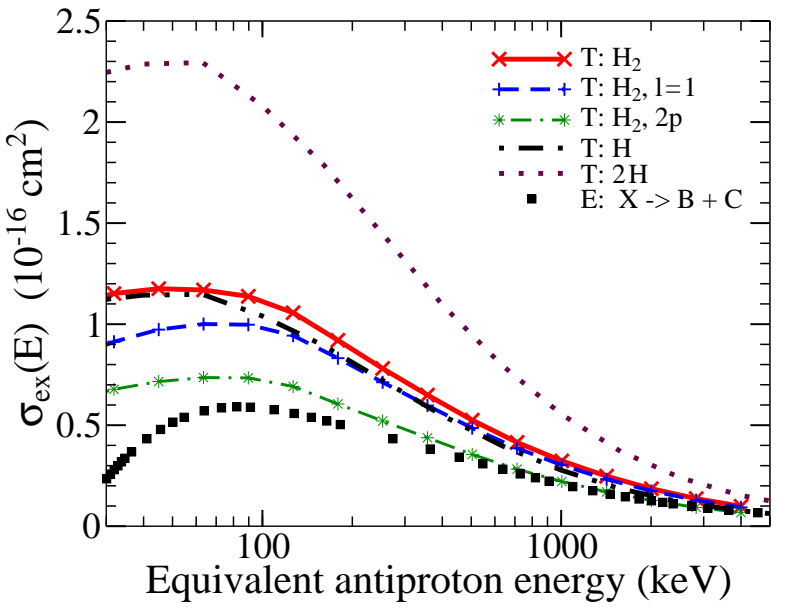

Fig. 2 Excitation cross section. Theory. $\mathrm{H}_{2}$ : solid curve, total; dashed curve, p states; dash-dotted curve, 2p. H: dash-doublydotted curve, total; dotted curve, two times total. Experiment. $\mathrm{H}_{2}$ : squares, $e^{-}: \mathrm{X} \rightarrow \mathrm{B}+\mathrm{C}$, Liu et al. 8. (See text)

satisfyingly. For smaller $E$, however, it follows rather the curve for twice the hydrogen atom. This behavior may originate from an insufficient description of the continuum (cf. [1]).

For high energies the present cross sections for $p$ and $\bar{p}$ are practically the same as can be expected in the validity regime of the first Born approximation which is independent of the sign of the projectile charge. For these energies the experimental results for $\bar{p}$ are, however, larger than those for $p$ by Rudd et al. This may originate from different normalization procedures. On the other hand, the present $p$ results agree with the experimental data in the whole energy range considered here; confirming the applicability of the used method even for $p$ collisions.
Excitation The results for excitation are presented in Fig. 2. A comparison of molecular with atomic hydrogen shows clearly that the simplified picture of $\mathrm{H}_{2}$ behaving basically like two $\mathrm{H}$ atoms is not senseful in the present context. Remarkably, the excitation cross section for one $\mathrm{H}$ atom is practically the same as for $\mathrm{H}_{2}$. It can also be seen that transitions into $\mathrm{p}(l=1)$ states (of the model potential) are by far the most prominent excitations. In particular, the differential cross section for the first excited $2 \mathrm{p}$ state is of the order of $2 / 3$ of the total excitation cross section. In the case of $\mathrm{H}_{2}$ excitation by $\bar{p}$ neither experimental nor other theoretical results exist in the literature to the authors' knowledge. Therefore, the present results for the excitation into the $2 p$ state are compared in Fig. 2 with experimental electron-collision results by Liu et al. 8 for excitations from the $\mathrm{H}_{2}$ ground state $\mathrm{X}$ into the energetically-lowest dipole-allowed states labeled with $\mathrm{B}$ and $\mathrm{C}$ which are orientationally averaged [12. The present results for $\bar{p}$ impact agree nicely with the experiment as expected at high energies. However, experimental excitation data for $\bar{p}$ impact would be valuable in order to judge the quality of the results at energies below the maximum at $E \approx 50 \mathrm{keV}$.

Electron-energy spectra In Fig. 3 electron-energy spectra for $\bar{p}$ and $p$ collisions with $\mathrm{H}_{2}$ are presented for $E=48 \mathrm{keV}$. The comparison of the $\bar{p}$ and $p$ calculations reveals different slopes for both curves. The most striking difference, however, is the peak in the case of $p$ impact at an electron energy $\epsilon$ which corresponds to the velocity of the proton whereas the $\bar{p}$ curve is lowered around this $\epsilon$. A comparison of the $p$ curve with measured data by Gealy et al. 3] yields good agreement except for the mentioned peak. The area under the maximum (hatched) and therefore the difference between the present and the experimental curve corresponds to the cross section for electron capture which is excluded in the experimental data.

Stopping power Using the differential information on ionization and excitation discussed so far it is possible to determine the electronic energy-loss cross section also referred to as stopping power $S$. In Fig. 4 the present results for $\bar{p}+\mathrm{H}_{2}$ are compared with experimental data by Lodi Rizzini et al. 9]. Also, data for negative muon $\left(\mu^{-}\right)$impact is given measured by Hauser et al. 4. Except for deviations at small velocities due to the mass dependence of the nuclear stopping power the results for $\bar{p}$ and $\mu^{-}$should be the same. The obvious discrepancies $(\approx 20 \%)$ between $\bar{p}$ and $\mu^{-}$indicate the experimental difficulties. The present results are for $E>200 \mathrm{keV}$ in good agreement with the $\mu^{-}$ 


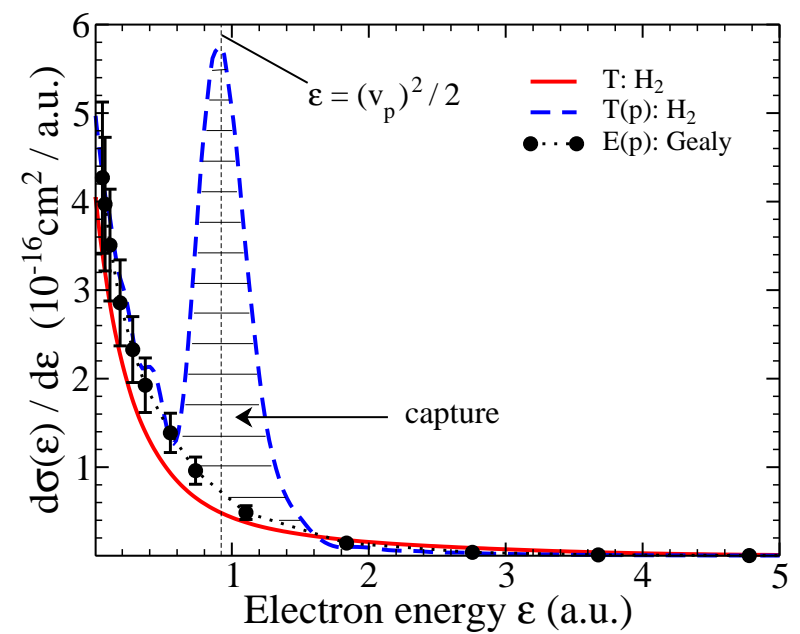

Fig. 3 Electron-energy spectra for $E=48 \mathrm{keV}$. Theory: solid curve, $\bar{p}$; dashed curve, $p$. Experiment: dots, Gealy et al. [3].

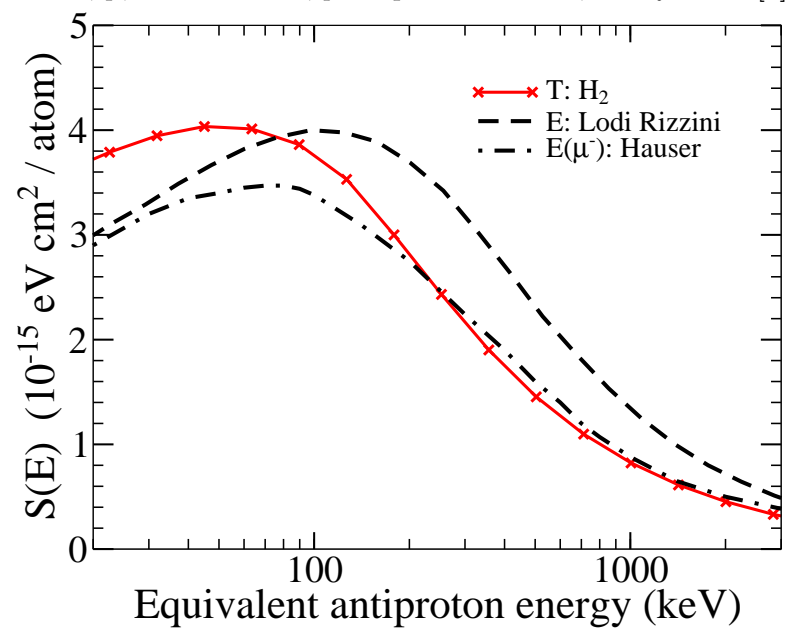

Fig. 4 Electronic stopping power for $\mathrm{H}_{2}$. Theory: solid curve, $\bar{p}$. Experiment: dashed curve, $\bar{p}$, Lodi Rizzini et al. [9]; dashdotted curve, $\mu^{-}$, Hauser et al. 4].

data. The ionization cross sections [1] used as input for the analysis in [4] were, however, found to be erroneous for $E<200 \mathrm{keV}$ [5,6]. On the other hand, the slope of the present curve for $E>100 \mathrm{keV}$ is remarkably similar to that of the experimental $\bar{p}$ curve but for $E>160$ $\mathrm{keV}$. This means that there seems to be a scaling of $E$ by a factor of $\approx 1.6$ between the present and the experimental $\bar{p}$ data. The stopping power for $\bar{p}+\mathrm{H}_{2}$ is currently investigated in detail in order to clarify this issue.

\section{Conclusion}

The present results obtained with a model potential are meaningful for ionization, excitation, and even for differential excitation and electron spectra for energies above the maximum around $60 \mathrm{keV}$. This opens up the possibility to further analyze the data in order to obtain doubly-differential cross sections. But also - as has been done here - to extract quantities like the stopping power for $\bar{p}+\mathrm{H}_{2}$ collisions.

The differences for ionization between experiment and theory at low $\bar{p}$ energies as well as between experiments for $\bar{p}$ and $p$ impact at high energies and the lack of measurements of excitation obviously show the need for new experimental $\bar{p}+\mathrm{H}_{2}$ data which may be available in the near future [6]. However, also an improvement of the theoretical description is necessary to account for the expected increase of importance of molecular (e.g., charge asymmetry, dissociation) and two-electron effects for low-energy impacts $E<100 \mathrm{keV}$ which are already indicated by the present findings. Additionally, the present stopping power results for $E>100 \mathrm{keV}$ agree with the experimental data for $\mu^{-}$but disagree for $\bar{p}$ making further investigation of $S(E)$ necessary.

Acknowledgements The authors are pleased to acknowledge discussions of the experimental results with Professors H. Knudsen and F. Kottmann. This work was supported by BMBF (FLAIR Horizon) and Stifterverband für die deutsche Wissenschaft.

\section{References}

1. Andersen, L.H. et al.: Non-dissociative and dissociative ionisation of $\mathrm{H}_{2}$ by $50-2000 \mathrm{keV}$ antiprotons. J. Phys. B 23, L395 (1990)

2. Ermolaev, A.M.: The ratio $R_{i}$ for nondissociative ionization of molecular hydrogen by antiproton/proton impact. Hyperfine Interact. 76, 335 (1993)

3. Gealy, M.W. et al.: Energy and angular distributions of electrons from ion impact on atomic and molecular hydrogen. I. 20-114-keV H ${ }^{+}+\mathrm{H}_{2}$. Phys. Rev. A 51, 2247 (1995)

4. Hauser, P. et al.: Slowing down of negative muons in gaseous $\mathrm{H}_{2}$ and determination of the stopping power. In: L.A. Schaller (eds.) Muonic atoms and molecules, p. 235 (1993)

5. Hvelplund, P. et al.: Ionization of helium and molecular hydrogen by slow antiprotons. J. Phys. B 27, 925 (1994)

6. Knudsen, H.: private communication (2008)

7. Knudsen, H. et al.: Ionization of helium and argon by very slow antiproton impact. Phys. Rev. Lett. 101, 043201 (2008)

8. Liu, X. et al.: Electron-impact excitation and emission cross sections of the $\mathrm{H}_{2}$ Lyman and Werner systems. J. Geophys. Res. 103, 26739 (1998)

9. Lodi Rizzini, E. et al.: Barkas effect for antiproton stopping in $\mathrm{H}_{2}$. Phys. Rev. Lett. 89, 183201 (2002)

10. Lühr, A., Saenz, A.: Antiproton and proton collisions with the alkali-metal atoms $\mathrm{Li}, \mathrm{Na}$, and K. Phys. Rev. A $\mathbf{7 7}$, 052713 (2008)

11. Lühr, A., Saenz, A.: Antiproton collisions with molecular hydrogen. Phys. Rev. A 78, 032708 (2008)

12. Lühr, A. et al.: Parameter-free one-center model potential for an effective one-electron description of molecular hydrogen. Phys. Rev. A 78, 042510 (2008)

13. Lühr, A. et al.: Interaction of antiprotons with Rb atoms and a comparison of antiproton stopping powers of the atoms $\mathrm{H}$, Li, Na, K, and Rb Hyperfine Interac. (2008) 
14. Rudd, M.E. et al.: Cross sections for ionization of gases by $5-4000-\mathrm{keV}$ protons and for electron capture by $5-150-\mathrm{keV}$ protons. Phys. Rev. A 28, 3244 (1983); Electron production in proton collisions: total cross sections. Rev. Mod. Phys. $\mathbf{5 7}, 965$ (1985)

15. Shah, M.B. et al.: Ionisation of $\mathrm{H}_{2}$ by fast protons and multiply charged ions of He, Li, C, N and O. J. Phys. B 15, 3441 (1982); Electron capture and ionisation in collisions of slow $\mathrm{H}^{+}$and $\mathrm{He}^{2+}$ ions with helium. J. Phys. B 22, 3037 (1989)

16. Vanne, Y.V., Saenz, A.: Ionization of $\mathrm{H}_{2}$ in intense ultrashort laser pulses: parallel versus perpendicular orientation. J. Mod. Opt. 55, 2665 (2008) 